\title{
Point of care ultrasonography (POCUS) in diagnosing ruptured ectopic pregnancy
}

\author{
Sarah K. Kennedy and Julie L. Welch
}

A 28-year female presented to the emergency department (ED) for 1 day of severe abdominal pain, vaginal bleeding, and syncope. Last menstrual period was unknown. Vital signs included a heart rate of $80 \mathrm{bpm}$ and blood pressure of $73 / 46 \mathrm{mmHg}$. She was moaning in distress and had diffuse tenderness with guarding and distention. On pelvic exam there was scant blood, a closed os, and adnexal tenderness. A bedside ED ultrasound revealed free fluid in the abdomen, no intrauterine pregnancy, and a heterogenous mass in the adnexa, concerning for a ruptured ectopic pregnancy with hemoperitoneum (Images $1-3$ ). The OB/GYN service was consulted. Urine pregnancy test was positive and hemoglobin was $7 \mathrm{~g} / \mathrm{dl}$. After resuscitation for hemorrhagic shock, she was taken for an emergent exploratory laparoscopy. Findings confirmed a $3.5 \mathrm{~cm}$ ruptured ectopic pregnancy with hemoperitoneum $(1.51 \mathrm{blood})$. A left salpingectomy was performed and she recovered uneventfully.

This is the authors' manuscript of the work published in final form as:

Kennedy, S. K., \& Welch, J. (2019). Point of care ultrasonography (POCUS) in diagnosing ruptured ectopic pregnancy. Visual Journal of Emergency Medicine, 16, 100606. 


\section{References}

1. Stein J.C., Wang R., Adler N., et al: Emergency physician ultrasonography for evaluating patients at risk for ectopic pregnancy: a meta-analysis. Ann Emerg Med 2010; 56: pp. 674-683

2. Moore C., Todd W.M., et al: Free fluid in Morison's pouch on bedside ultrasound predicts need for operative intervention in suspected ectopic pregnancy. Acad Emerg Med 2007; 14: pp. $755-758$ 


\section{Figures}

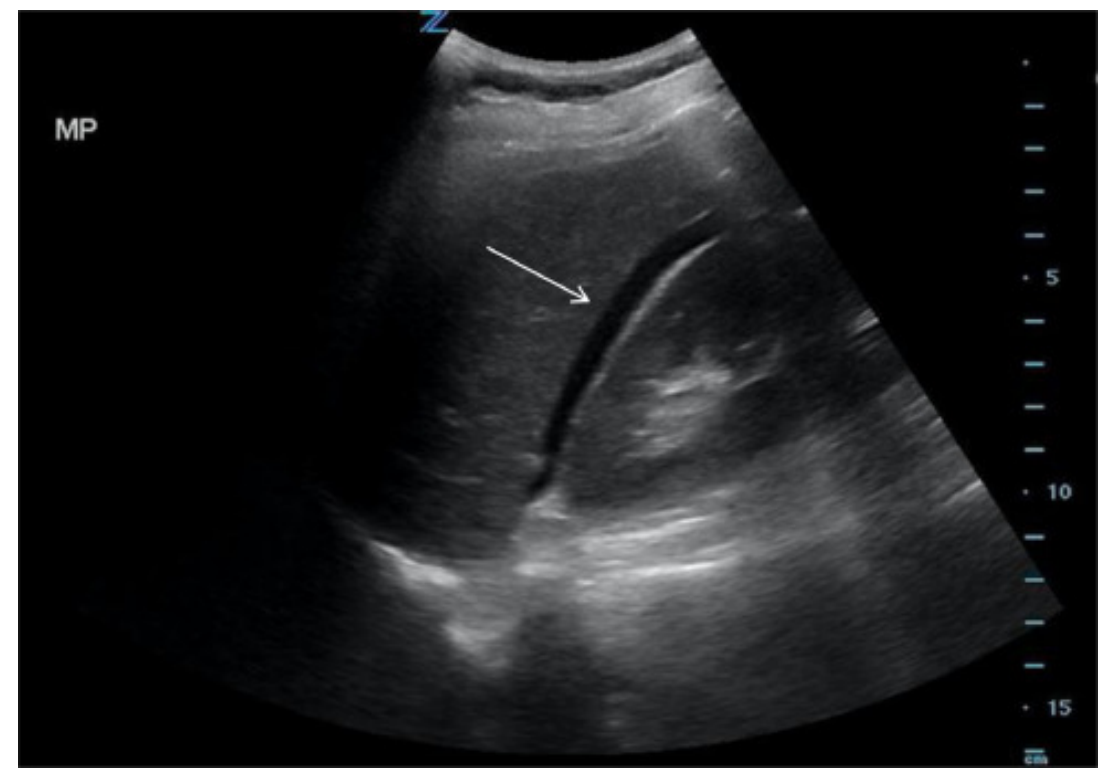

Image 1

Transabdominal ultrasound image of hepatorenal recess (Morison's pouch) demonstrating free intraperitoneal fluid stripe (arrow).

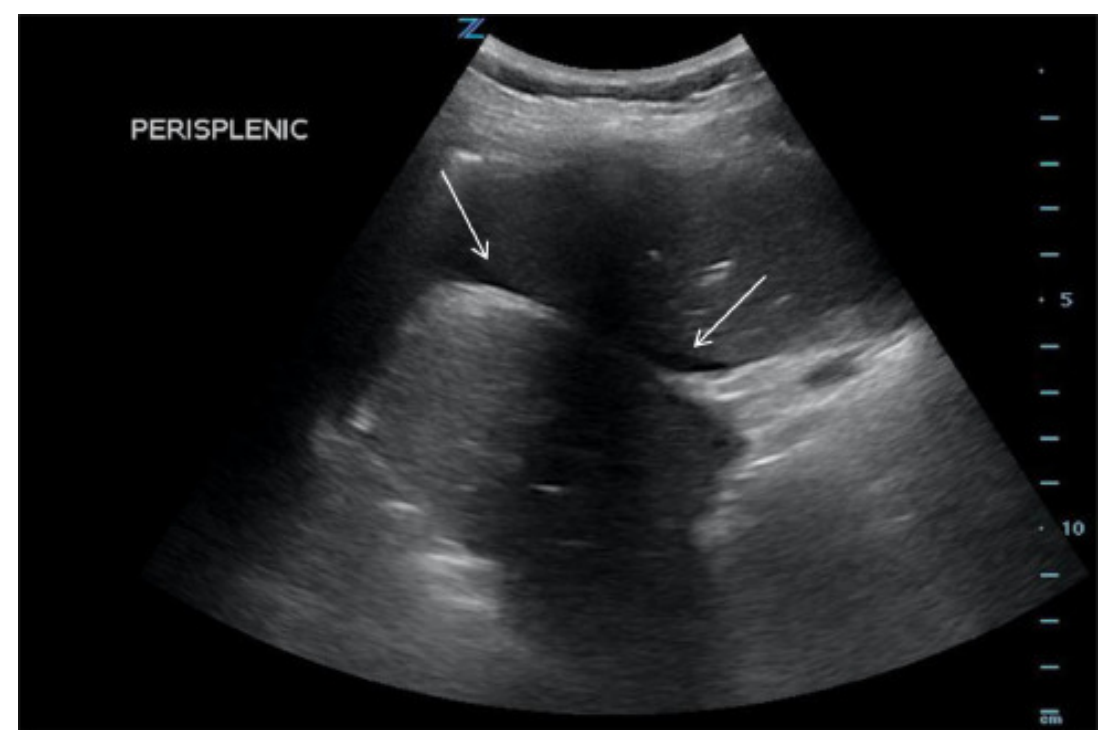

Image 2

Transabdominal ultrasound image of perisplenic space demonstrating free intraperitoneal fluid stripe (arrows). 


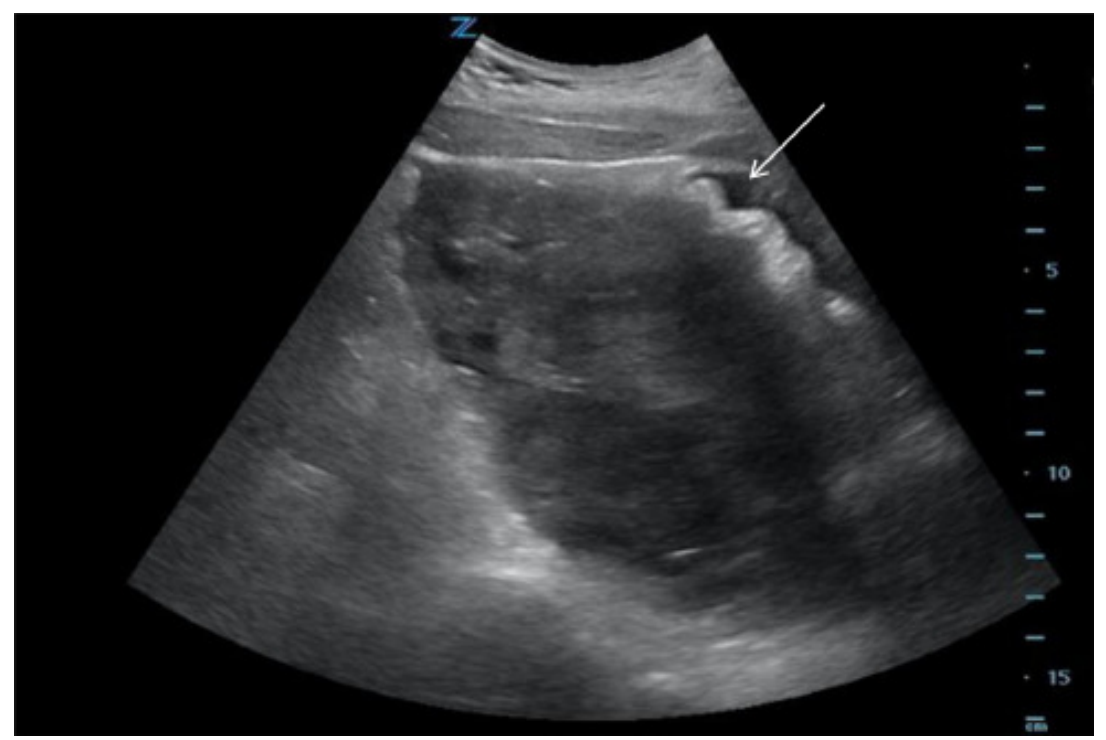

Image 3

Transabdominal ultrasound image of pelvis (left adnexal region) demonstrating a heterogeneous collection with free fluid (arrow) and clot. 